CME. Therapie der chronischen Hepatitis C

Schaffstein, Stella ; Rampini, Silvana K ; Müllhaupt, Beat

DOI: https://doi.org/10.1024/1661-8157/a001618

Other titles: Therapy of chronic hepatitis C

Posted at the Zurich Open Repository and Archive, University of Zurich ZORA URL: https://doi.org/10.5167/uzh-102672

Journal Article

Originally published at:

Schaffstein, Stella; Rampini, Silvana K; Müllhaupt, Beat (2014). CME. Therapie der chronischen Hepatitis C. Praxis, 103(8):423-436.

DOI: https://doi.org/10.1024/1661-8157/a001618 


\section{Therapie der chronischen Hepatitis C}

Das Hepatitis C Virus (HCV) ist ein kleines RNA-Virus aus der Familie der Flaviviridae, bei welchem 7 verschiedene Genotypen unterschieden werden. Genotyp 1 ist weltweit am häufigsten nachweisbar. Genotyp 2 ist vor allem im Mittelmeerraum, Genotyp 4 vornehmlich in Ägypten anzutreffen. Bei i.v.Drogenkonsumenten findet sich gehäuft der Genotyp 3. Genotyp 5 und 6 kommen insgesamt selten vor, ebenso wie der erst kürzlich neu beschriebene Genotyp 7, welcher in Kanada und Belgien identifiziert wurde [1-2].

Die Übertragung von HCV erfolgt auf parenteralem Wege. Neuinfektionen sind heutzutage hauptsächlich auf i.v.-Drogenkonsum zurückzuführen. Eine Übertragung durch Blutprodukte ist seit Einführung von Screeningtests von Blutprodukten auf HCV-RNA selten geworden. Das Risiko einer perinatalen oder sexuellen Übertragung ist im Gegensatz zur Hepatitis-B-Infektion gering (bei vaginalem Geschlechtsverkehr ca. 0-0.7\% pro Jahr, höher allerdings bei Analverkehr, vertikales Übertragungsrisiko ca. 5\%) [3-4].

Eine Infektion mit HCV ist eine der häufigsten Ursachen für eine chronische Lebererkrankung und Indikation für eine Lebertransplantation. In nur 20\%-40\% der Fälle verläuft die Infektion akut und heilt wieder aus. In 60\%-80\% kommt es zur chronischen Hepatitis, d.h. HCV-RNA ist nach 6 Monaten noch immer nachweisbar. Von den chronisch Infizierten entwickeln 5-20\% der Fälle innerhalb von zwei Jahrzehnten eine Leberzirrhose und von diesen wiederum 1-6\% pro Jahr ein hepatozelluläres Karzinom (HCC). Weltweit sind ca. 120-200 Millionen Menschen mit HCV infiziert. In der Schweiz wird die Zahl chronisch Infizierter auf ca. 1-1.5\% geschätzt, wovon 50\% auf den Genotyp 1 zurückzuführen sind [5].

Die Therapieoptionen der chronischen Hepatitis C sind seit den letzten Jahren in grossem Umbruch, insbesondere beim schwierig zu behandelnden Genotyp 1. Grundsätzlich sollte bei allen Patienten die Behandlungsindikation überprüft werden, oft sind aber Komorbiditäten oder Nebenwirkungen ein limitierender Faktor. Bei Patienten mit Genotyp 1 ist derzeitiger Standard eine Kombinationstherapie aus einem oralen Proteaseinhibitor als direkt antivirales Medikament zusammen mit pegyliertem Interferon-alfa (PEG-IFN- $\alpha$ ) und Ribavirin (RBV) (Tripletherapie). Für Patienten mit Genotyp 2, 3, 4, 5 und 6 sind derzeit ausserhalb von klinischen Studien noch keine direkt antiviralen Medikamente zugelassen. Der bisherige Standard bei diesen Genotypen ist weiterhin noch eine Kombinationstherapie mit PEG-IFN- $\alpha$ und RBV [1]. Aufgrund diverser Kontraindikationen für Interferon- $\alpha$ bleiben hier aber leider noch immer viele Patienten unbehandelt. Durch neue direktantivirale Medikamente sind nicht nur neue Therapiekonzepte entstanden, sondern es ist die Hoffnung gewachsen, diese teils schwerwiegende Infektionskrankheit in Kürze gezielter, vereinfachter, erfolgreicher und nebenwirkungsarmer behandeln zu können.

In diesem CME gehen wir auf die Entscheidung zur Therapie sowie alle Aspekte vor, während und nach der Therapie einer Hepatitis $\mathrm{C}$ ein. 


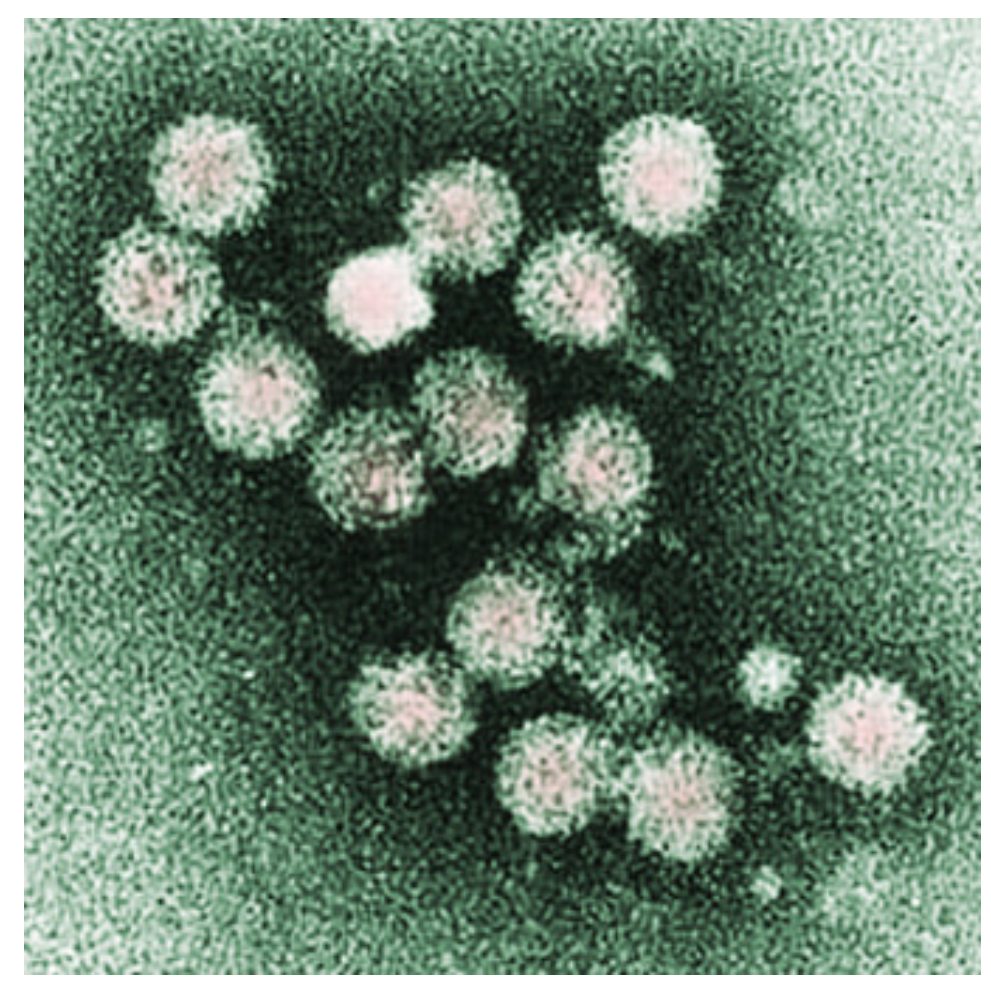

\section{Legende}

Hepatitis C Viren im Elektronenmikroskop. Quelle: flickr.com. AJC1, 02.02.2012, unverändert übernommen.

\section{Im Artikel verwendete Abkürzungen}

AFP
ALT
AST
BMI
BOC
DAA
GGT
HAV
HBV
HCV
HCC
HIV
IFN- $\alpha$
PEG-INF- $\alpha$
INR
MRI
NSAR
PCR
RBV
RNA
TPO
TPV
TSH

Alpha-Fetoprotein

Alanin-Aminotransferase

Aspartat-Aminotransferase

Body Mass Index

Boceprevir

direct antiviral agents

Gamma Glutamyl Aminotransferase

Hepatitis A Virus

Hepatitis B Virus

Hepatitis C Virus

Hepatocelluläres Carcinom

Humanes Immundefizienz Virus

Interferon alfa

Pegyliertes Interferon alfa

International Normalized Ratio

Magnet Resonanz Imaging

Nichtsteroidale Antirheumatika

Polymerase chain reaction

Ribavirin

Ribonucleic Acid

Thyreoperoxidase

Telaprevir

Thyreoidea stimulierendes Hormon 


\section{Abklärungen vor Therapiebeginn}

Die Diagnose der Hepatitis $\mathrm{C}$ wird durch den Nachweis von anti-HCV-Antikörpern sowie HCV-RNA im Serum gestellt. Die Quantifizierung der HCV-RNA muss als Referenz zur Beurteilung des Therapieansprechens vor Therapiebeginn durchgeführt werden [1].

Sobald die Diagnose gestellt wurde, müssen zunächst Abklärungen erfolgen, welche das Ausmass der Lebererkrankung evaluieren. Hierzu gehören die Bestimmung der Transaminasen (AST, ALT), der GGT, alkalischen Phosphatase, Bilirubin, INR, Albumin und ein Blutbild sowie die Durchführung einer Abdomensonographie [2].

Zudem müssen andere Ursachen einer chronischen Lebererkrankung gesucht werden, wie eine KoInfektion mit HIV, Hepatitis A und B. Im Falle eines negativen HAV und HBV-Nachweises sollte zeitnah eine Impfung erfolgen [2].

Ausserdem müssen Risikofaktoren, die eine beschleunigte Progression zur Leberfibrose und -zirrhose bewirken, gezielt gesucht bzw. erfragt werden. Dies sind übermässiger Alkoholkonsum, Tabak- und Cannabiskonsum, Übergewicht (BMl $>25 \mathrm{~kg} / \mathrm{m} 2)$ mit evtl. Steatosis hepatis oder Steatohepatitis, Insulinresistenz, Glukosetoleranzstörung bzw. Diabetes mellitus sowie eine Immunsuppression. Da durch die gängigen Therapieschemata mit Interferon- $\alpha$ eine Autoimmunthyreoiditis ausgelöst werden kann, sollten vor Therapiebeginn auch TSH sowie die TPO-Antikörper bestimmt werden [2].

Schliesslich sind diverse Abklärungen, die den Therapieentscheid beeinflussen, notwendig. Die Bestimmung des Genotyps ist wichtig, um das Therapieschema festzulegen, da aktuell eine Infektion mit dem Genotyp 1 anders behandelt wird als eine Infektion mit den übrigen Genotypen. Der Nachweis eines genetischen Polymorphismus auf dem Chromosom 19, welcher für Interferon lambda3 (früher IL28B) kodiert, ist vor allem beim Genotyp 1 mit einem besseren Therapieansprechen assoziiert. Die Bestimmung dieses Polymorphismus hat allerdings keine Auswirkung auf den Therapieentscheid, sondern nur auf die Prognose [2].

Die Bestimmung des Schweregrades der Lebererkrankung ist notwendig, um die Wichtigkeit eines raschen Therapiebeginns sowie die Prognose zum Therapieansprechen abschätzen zu können. Hierzu dient einerseits die Durchführung einer Leberbiopsie. Diese sollte bei Vorliegen von mehreren potentiellen Auslösern einer chronischen Lebererkrankung sowie bei zusätzlich vorliegenden Risikofaktoren mit beschleunigter Fibroseentwicklung durchgeführt werden. Durch die Histologie erfolgt dann ein Grading (entzündliche Aktivität) und ein Staging (Fibrosegrad). Die Quantifizierung der entzündlichen Aktivität und Fibrose erfolgt durch verschiedene Scores, z.B. der METAVIR-Score (F0= keine Fibrose, bis F4=Zirrhose) oder der Ishak-Score (von F0 bis F6). Alternativ kann als nichtinvasive Methode zum Fibrosestaging eine Ultraschall-Elastographie (Fibroscan®) durchgeführt werden. Zu beachten ist bei dieser Untersuchung allerdings, dass bei Übergewicht die Messung häufig nicht gelingt. Beim Nachweis einer Zirrhose oder bei sehr wahrscheinlich vorliegender Zirrhose muss eine endoskopische Suche nach Ösophagus- und Fundusvarizen sowie eine regelmässige (alle 6 Monate) Überwachung mittels Ultraschall und Bestimmung des $\alpha$-Fetoproteins zur Früherfassung eins hepatozellulären Karzinoms (HCC) erfolgen [1-2].

Je nach Symptomkonstellation müssen bei einer chronischen Hepatitis $C$ auch extrahepatische Erkrankungen gesucht werden. Mit HCV assoziierte Erkrankungen sind beispielsweise Kryoglobulinämie, Lichen ruber planus, Porphyria cutanea tarda, membranöse Glomerulonephritis sowie gewisse Formen der Non-Hodgkin-Lymphome [2].

Bevor der Entscheid zum Therapiebeginn gefällt wird, muss dem Patienten erläutert werden, dass ein konsequenter Therapieplan mit relativ streng vorgeschriebenen Nachkontrollen und regelmässiger Medikamenteneinahme notwendig ist. Dies gilt insbesondere bei der Behandlung des Genotyp 1 mit Tripletherapie. Auf mögliche Nebenwirkungen (Details siehe unten) sollte bereits vorab hingewiesen werden, damit sich der Patient darauf einstellen kann. Auch die Behandlung bzw. Minimierung negativer Kofaktoren wie Alkohol-, Zigaretten-/Kannabiskonsum und Gewichtsreduktion sollten schon vor Therapiebeginn besprochen werden.

Schliesslich sollte jeder Patient mit Diagnose einer Hepatitis C über die Transmissionsfaktoren dieser Erkrankung aufgeklärt werden, um eine Übertragung auf andere Personen zu vermeiden. So sollten mit HCV Infizierte keine potentiell mit Blut kontaminierten Gegenstände mit anderen Personen teilen, wie zum Beispiel Rasierer, Zahnbürsten oder Nadeln bei intravenösem Drogenkonsum. Hingegen ist die Verwendung von Kondomen nur bei Promiskuität und analem Geschlechtsverkehr empfohlen. Bei einer langjährigen stabilen sexuellen Beziehung ist aufgrund des geringen Risikos einer sexuellen Übertragung eine Verwendung von Kondomen nicht notwendig. Bei HCV Infizierten Müttern ist kein Kaiserschnitt notwendig und die Mutter darf ihr Kind stillen. Frühestens 18-24 Monate nach Geburt 
sollte das Kind auf Hepatitis $\mathrm{C}$ getestet werden, da maternale anti-HCV-Antikörper noch mehrere Monate nach Geburt im Blut des Kindes persistieren [2; 4].

\section{田}

Grundsätzlich sollten alle Patienten mit einer chronischen Hepatitis C hinsichtlich der Durchführbarkeit und Notwendigkeit einer Therapie evaluiert werden. Ein rascher Therapiebeginn sollte bei Patienten mit fortgeschrittener Fibrose (METAVIR Score F3-F4) erfolgen. Bei Patienten mit moderater Fibrose (METAVIR Score F2) sollte eine Therapie empfohlen werden. Bei Patienten mit milder Lebererkrankung muss zusammen mit dem Patienten Nutzen und Risiko hinsichtlich Therapienebenwirkungen abgewogen werden [1-2]. In Anbetracht der in Kürze zu erwartenden neuen, vereinfachten, hochwirksamen und nebenwirkungsärmeren Therapieoptionen ist hier eine abwartende Haltung zu bevorzugen.

Kontraindikationen sind insbesondere bei Interferon-enthaltenden Therapieschemata zu beachten. Diese sind in Tabelle 1 aufgeführt. Ein persistierender Alkoholkonsum ist zwar ungünstig, stellt aber keine Kontraindikation dar.

\section{Absolute Kontraindikationen}

\section{Unkontrollierte Depression}

Akute Suizidalität

Psychose

Unkontrollierte Epilepsie

Aktive unkontrollierte Autoimmunerkrankung

Dekompensierte Leberzirrhose (Child Pugh B7 und mehr)

Schwere unkontrollierte Hypertonie, Herzinsuffizienz, Diabetes mellitus oder COPD

Malignom mit ungünstiger Prognose

Schwangerschaft und Stillzeit

\section{Relative Kontraindikationen}

Frühere schwere Depression oder Suizidalität in der Vorgeschichte

Anämie (Hämoglobin $<13 \mathrm{~g} / \mathrm{l}$ beim Mann, $<12 \mathrm{~g} / \mathrm{l}$ bei der Frau)

Neutropenie $<1500 / \mathrm{ul}$

Thrombopenie $<90.000 /$ ul

Leberzirrhose Child A mit signifikanter portaler Hypertonie (Thrombozyten $<90^{\prime} 000 / u$ und Albumin $<35 \mathrm{~g} / \mathrm{l})$

Signifikante koronare Herzkrankheit

Unbehandelte Schilddrüsenerkrankung

Tab. 1: Absolute und Relative Kontraindikationen bei Interferon-enthaltenden Therapieschemata [2]. 


\section{If Therapiealgorithmus}

Das primäre Ziel der HCV-Therapie ist die Eliminierung des HC-Virus, sodass die HCV-RNA anhaltend nicht mehr im Serum mittels der gängigen PCR-Assays nachweisbar ist. Man spricht hier von "sustained virological response“ (SVR), d.h. HCV-RNA ist 12 (SVR 12) oder 24 Wochen (SVR 24) nach Therapieende nicht mehr nachweisbar.

Die Therapiedauer ist wesentlich abhängig vom Therapieansprechen. Hier hat sich die sogenannte „response guided therapy“ durchgesetzt. Die Therapiedauer wird dann entsprechend dem Therapieansprechen individuell verkürzt oder verlängert. Definiert wird das Therapieansprechen durch das Ausmass der Reduktion nachweisbarer HCV-RNA im Serum zu einem bestimmten Zeitpunkt während der Therapie. Die verschiedenen Definitionen sind in Tabelle 2 aufgeführt. Zur Definierung des Therapieansprechens muss die HCV-RNA nach 4, 12 und 24 Wochen Therapiedauer, am Ende der Therapie sowie 12 oder 24 Wochen nach Ende der Therapie gemessen werden, bei Therapie mit Boceprevir zusätzlich nach 8 Wochen Therapiedauer [1-2].

\begin{tabular}{|c|c|}
\hline Rapid virological response (RVR) & Nicht nachweisbare HCV RNA nach 4 Wochen \\
\hline Extended RVR (eRVR) & $\begin{array}{l}\text { Nicht nachweisbare HCV RNA nach } 4 \text { und } 12^{1} \\
\text { Wochen }\end{array}$ \\
\hline RVR8 & Nicht nachweisbare HCV RNA nach 8 Wochen² \\
\hline Early virological response (EVR) & >2 log Stufen Abfall der HCV RNA nach 12 Wochen \\
\hline Complete EVR (cEVR) & Nicht nachweisbare HCV RNA nach 12 Wochen \\
\hline Partial EVR (pEVR) & $\begin{array}{l}>2 \text { log Stufen Abfall aber noch nachweisbare HCV } \\
\text { RNA nach } 12 \text { Wochen }\end{array}$ \\
\hline Delayed virological response $(\mathrm{RVR})^{3}$ & $\begin{array}{l}>2 \text { log Stufen Abfall aber noch nachweisbare HCV } \\
\text { RNA nach } 12 \text { Wochen, nicht nachweisbar nach } 24 \\
\text { Wochen }\end{array}$ \\
\hline Partial response (PR) & $\begin{array}{l}>2 \text { log Stufen Abfall der HCV RNA nach } 12 \text { Wochen } \\
\text { aber nachweisbar nach } 12 \text { und } 24 \text { Wochen }\end{array}$ \\
\hline Null response (NR) & $<2$ log Stufen Abfall der HCV RNA nach 12 Wochen \\
\hline Breakthrough (BT) & Wiederauftreten von HCV RNA während Therapie \\
\hline Relapse & $\begin{array}{l}\text { HCV RNA am Therapieende nicht nachweisbar aber } \\
\text { nach } 24 \text { Wochen Therapieende wieder nachweisbar }\end{array}$ \\
\hline Sustained virological response (SVR) & $\begin{array}{l}\text { Nicht nachweisbare HCV RNA } 12 \text { (SVR 12) oder } 24 \\
\text { (SVR 24) Wochen nach Therapieende }\end{array}$ \\
\hline
\end{tabular}

Tab. 2. Definitionen des Therapieansprechens

${ }^{1}$ Bezieht sich auf Tripletherapie mit Telaprevir

${ }^{2}$ Bezieht sich auf Tripletherapie mit Boceprevir mit einer 4 wöchigen Lead-in Phase mit PEG-INF- $\alpha$ und RBV

${ }^{3}$ Früher als slow virological response bezeichnet

Das Therapieschema mit entsprechender Wahl der antiviralen Medikamente wird anhand verschiedener Kriterien gewählt. Hierzu gehört einerseits der nachgewiesene Genotyp. Eine Infektion mit dem Genotyp 1 wird anders behandelt als eine Infektion mit den übrigen Genotypen. Andererseits muss beachtet werden, ob der Patient bereits in der Vorgeschichte eine erfolglose oder nur teils erfolgreiche Therapie durchlaufen hat, respektive ob der Patient Therapie-naiv, ein Null-Responder, ein Partieller-Responder oder ein Relapser ist. Zudem ist es für das Therapieschema von Relevanz, ob eine Leberzirrhose vorliegt oder nicht.

Der frühere Behandlungsstandard der chronischen Hepatitis $C$ war eine Kombinationstherapie mit pegyliertem Interferon- $\alpha 2 a$ oder $2 b$ zusammen mit Ribavirin. Ein wesentlicher Unterschied zwischen den beiden PEG-INF- $\alpha$ hinsichtlich Therapieverträglichkeit oder -ansprechen konnte nicht nachgewiesen werden. Beim Genotyp 2, 3, 4, 5 und 6 ist diese Therapie derzeit immer noch Standardtherapie. Die SVR-Raten unter dieser Kombinationstherapie sind auch relativ hoch mit $80 \%$. Beim Genotyp 1 und 4 sind sie hingegen deutlich niedriger mit 40-50\% [5]. 
Mittlerweile ist eine grosse Anzahl von neuen direkt-antiviralen Medikamenten, sogenannten „direct antiviral agents" (DAA), in verschiedenen Stadien von präklinischen und klinischen Studien in Entwicklung.

Seit Anfang 2012 sind in der Schweiz die antiviralen Medikamente Telaprevir (Incivo®) und Boceprevir

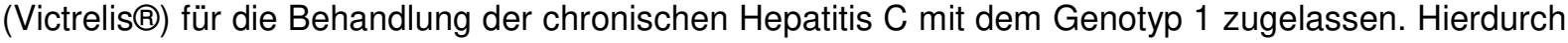
konnten die SVR-Raten um $25 \%$ auf bis zu $75 \%$ bei Therapie-naiven gesteigert werden, bei Relapsern auf $>80 \%$, bei Partiell-Respondern auf $50 \%$ und bei vorherigen Null-Respondern auf $30 \%$ [5]. Deshalb ist der heutige Therapiestandard beim Genotyp 1 eine Tripletherapie aus Telaprevir oder Boceprevir zusammen mit PEG-INF- $\alpha$ und RBV.

Aufgrund der raschen Entwicklung von Resistenzen müssen Telaprevir und Boceprevir immer in Kombination mit PEG-INF- $\alpha$ und RBV verabreicht werden.

Eine Zulassung neuer DAAs ohne Notwendigkeit einer Kombination mit Interferon-a, welche für alle Genotypen gleichsam wirksam sind, ist absehbar. Dies wird die Behandlung der Hepatitis C wesentlich vereinfachen und somit grundsätzlich verändern.

Im Folgenden werden die unterschiedlichen Therapiealgorithmen einerseits beim Genotyp 2, 3, 4, 5 und 6 und andererseits beim Genotyp 1 sowie die neuen Therapieoptionen erläutert. Der erst neu beschriebene Genotyp 7 wird hier aufgrund der fehlenden Datenlage bewusst nicht erwähnt.

\section{Therapiealgorithmus bei Genotyp 2, 3, 4, 5 und 6}

Bei den Genotypen 2, 3, 4, 5 und 6 wird (abgesehen von klinischen Studien) derzeit noch das alt bewährte Therapieregime mit PEG-INF- $\alpha$ und RBV durchgeführt.

Pegyliertes Interferon- $\alpha$ 2a sollte in der Dosierung von $180 \mu \mathrm{g}$ einmal pro Woche subkutan appliziert werden. Pegyliertes Interferon- $\alpha$ 2b muss gewichtsadaptiert in einer Dosis von 1,5 $\mu \mathrm{g} / \mathrm{kgKG}$ pro Woche verabreicht werden. Die Dosierung von RBV ist abhängig vom vorliegenden Genotyp. Bei den Genotypen 4-6 erfolgt die Gabe von 15 mg/kgKG pro Tag (oder 1000/1200 je nach Gewicht). Beim Genotyp 2 und 3 kann RBV in einer fixen Dosierung von $800 \mathrm{mg}$ pro Tag gegeben werden. Auch Patienten mit einem BMI von $>25 \mathrm{~m}^{2} / \mathrm{kg}$ oder mit Risikofaktoren, welche eine schlechtere Ansprechrate prognostizieren (Insulinresistenz, Metabolisches Syndrom, schwere Fibrose oder Leberzirrhose, höheres Alter) sollten eine gewichtsadaptierte Dosis von RBV erhalten [1-2].

Die Dosis von PEG-INF- $\alpha$ und RBV muss je nach Entwicklung von Nebenwirkungen ggf. reduziert werden (siehe Kapitel "Management während und nach Therapie").

Die Therapiedauer wird je nach Therapieansprechen individuell bestimmt. Hierzu wird die HCV-RNA nach 4, 12 und 24 Wochen Therapie gemessen. Ein Überblick verschafft hier Abbildung 1.

Bei Patienten mit Genotyp 2 und 3 mit rapid virological response (RVR) und niedrigem Baseline Viraload $(<400.000-800.000 \mathrm{IU} / \mathrm{ml})$ kann die Verkürzung der Therapiedauer auf 12-16 Wochen erwogen werden. Die Empfehlung einer Therapieverkürzung auf 12-16 Wochen gilt allerdings nicht für die Genotypen 4-6. Eine Verkürzung der Therapiedauer bei Genotyp 2 und 3 wird nicht empfohlen, wenn eine fortgeschrittene Fibrose, eine Zirrhose oder Kofaktoren mit ungünstiger Ansprechrate (Insulinresistenz, Metabolisches Syndrom) vorliegen. Patienten mit Genotyp 2 und 3 mit early virological response (EVR) oder delayed virological response (DVR) oder beim Vorliegen negativer Kofaktoren werden für 48 Wochen behandelt, vorausgesetzt die HCV-RNA ist nach 24 Wochen nicht mehr nachweisbar [1].

Beim Genotyp 5 und 6 liegen keine guten Daten für eine response guided therapy vor, eine Therapieverkürzung auf 24 Wochen wird aber diskutiert. Bei Genotyp 4 kann bei RVR und niedrigem Baseline Viraload (<400.000-800.000 IU/ml) eine Therapiedauer von 24 Wochen gewählt werden, bei erhöhtem Baseline Viraload und EVR wird hingegen eine Therapiedauer von 48 Wochen, bei DVR sogar von 72 Wochen empfohlen [1].

In gewissen Fällen ist ein Therapieabbruch indiziert. Hier gibt es klar definierte Stopp-Regeln [1-2]. Diese sind:

- HCV-RNA nach 12 Wochen $<2$ log Stufen UI/ml abgesunken

- HCV-RNA nach 24 Wochen noch nachweisbar

- schwere Nebenwirkungen wie schwere Depression, Neutropenie <500/ $\mu$, Thrombopenie $<25.000 / \mu$ l, entzündlicher Hepatitis-Schub mit Anstieg der Transaminasen über das 10-fache der Norm sowie schwere bakterielle Infektion 
Bei Null-Respondern sollte ein Therapieversuch mit neuen direkt-antiviralen Medikamenten im Rahmen klinischer Studien erwogen werden. Eine Zulassung neuer Medikamente ist bereits für 2014 geplant.

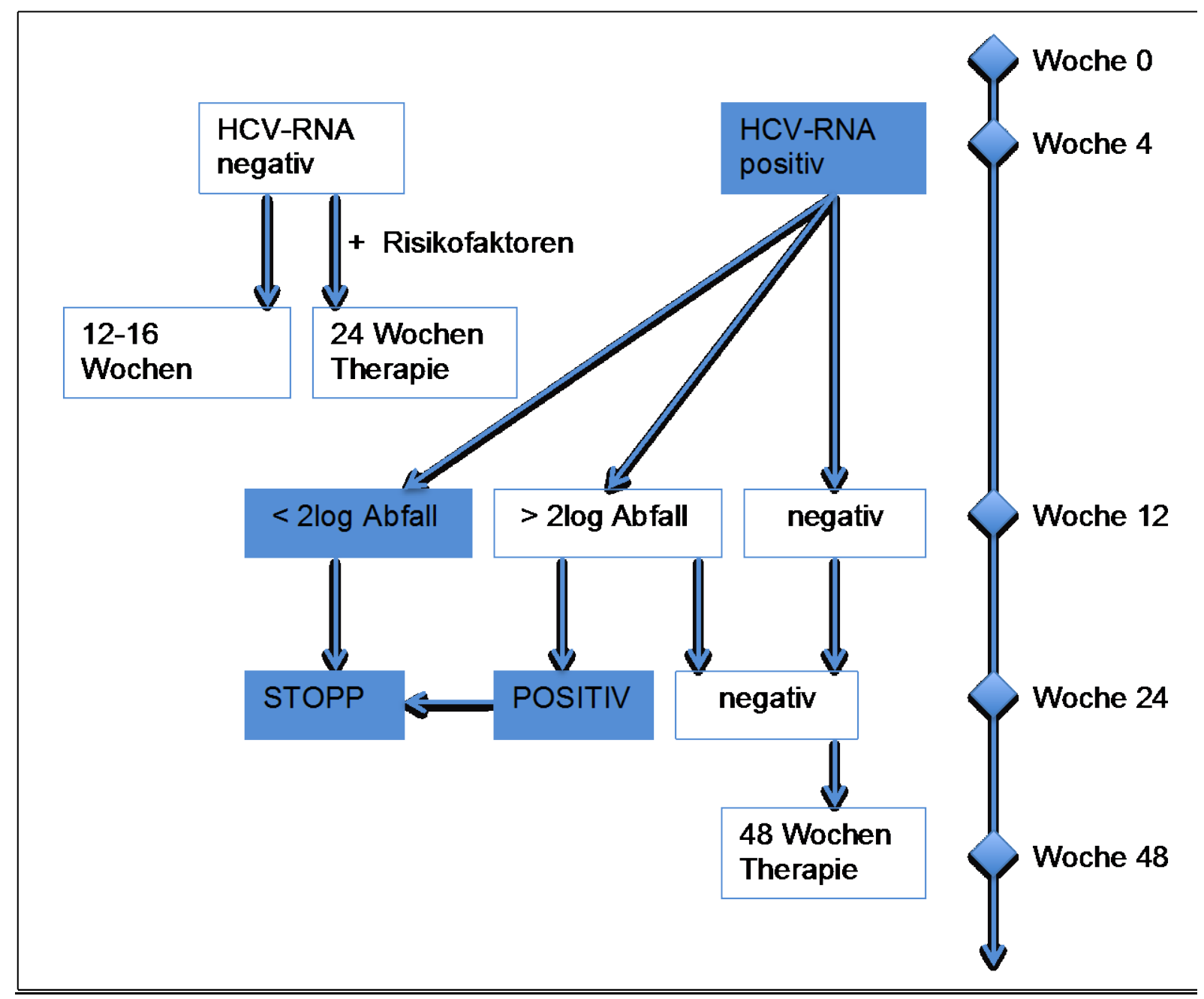

Abb.1 Response guided therapy bei Patienten mit Genotyp 2 und 3 (bei Genotyp 4, 5 und 6 gilt die Option der Therapieverkürzung auf 12-16 Wochen nicht)

\section{Therapiealgorithmus bei Genotyp 1}

Bei Infektion mit dem Genotyp 1 wird in der Regel zur Erhöhung der SVR-Rate eine sogenannte Tripletherapie empfohlen. Diese beinhaltet eine Kombination aus PEG-INF- $\alpha$, RBV sowie einem der NS3/4A-Proteaseinhibitoren der 1. Generation Telaprevir (TPV) oder Boceprevir (BOC). Diese Therapie ist deutlich wirksamer, allerdings ist auch die Anzahl und Ausprägung von Nebenwirkungen unter diesem Therapieregime höher im Vergleich zur alleinigen Therapie mit PEG-INF- $\alpha$ und RBV [5]. Die Therapieindikation muss entsprechend sorgfältig gestellt werden.

Die Empfehlung für eine Tripletherapie gilt sowohl bei Therapie-naiven Patienten als auch bei Patienten mit Relapse, Partiell- oder Null-Respondern. 
Bei Therapie-naiven Patienten mit sehr guten Baseline Parametern (HCV-RNA $<400.000 \mathrm{IU} / \mathrm{ml}$, Fehlen von fortgeschrittener Fibrose oder Zirrhose), welche eine RVR erreichen, kann eine 4-wöchige Lead-in-Phase mit PEG-INF- $\alpha$ und RBV erfolgen. Die Therapie kann dann ggf. ohne Hinzugabe von Telaprevir oder Boceprevir für 24 Wochen weitergeführt werden [5].

Bei bisherigen Null-Respondern unter PEG-INF- $\alpha$ und RBV, insbesondere bei Vorliegen einer Zirrhose muss der Versuch einer Tripletherapie kritisch evaluiert werden. Bei diesen Patienten sollte ein Einschluss in klinische Studien mit neuen DAAs oder Interferon-sparenden Regimes diskutiert werden bzw. auf die Zulassung neuerer DAAs gewartet werden.

Telaprevir muss in einer Dosis von $750 \mathrm{mg}$ alle 8 Stunden oder $1115 \mathrm{mg}$ alle 12 Stunden mit einer ca. $20 \mathrm{~g}$ Fett enthaltenden Mahlzeit eingenommen werden. Boceprevir muss in einer Dosis von $800 \mathrm{mg}$ alle 8 Stunden eingenommen werden. Die relativ strikte Dosierung ist wichtig, um die antivirale Wirkung des Medikamentes im Serum konstant zu halten und damit der Resistenzentwicklung entgegenzuwirken. Aufgrund der Tendenz zur raschen Resistenzentwicklung dürfen Telaprevir oder Boceprevir auch niemals ohne die Kombination mit PEG-INF- $\alpha$ und RBV verabreicht werden $[1 ; 5]$.

Die Dosierung von PEG-INF- $\alpha$ und RBV erfolgt in Anlehnung der in Studien verwendeten Dosierung. $[1 ; 5]$.

Die Therapieschemata mit Telaprevir einerseits und Boceprevir andererseits unterscheiden sich bezüglich Therapiedauer und Therapiebeginn. Telaprevir wird in der Regel zum Therapieanfang zusammen mit PEG-INF- $\alpha$ und RBV während 12 Wochen verabreicht. Die Therapiedauer wird bestimmt durch das virologische Ansprechen (eRVR) in Woche 4 und 12, den Fibrosegrad (Zirrhotiker vs. Nicht-Zirrhotiker) und das initiale Therapieansprechen bei Therapie-Erfahrenen. Im Gegensatz dazu wird bei Verwendung von Boceprevir immer eine 4-wöchige Lead-In-Phase mit PEG-INF- $\alpha$ und RBV durchgeführt, bevor mit Boceprevir begonnen wird. Die Therapiedauer wird dann durch das virologische Ansprechen (RVR8), den Fibrosegrad und das initiale Therapieansprechen bestimmt $[1 ; 5]$.

Die spezifischen Therapiealgorithmen von Telaprevir und Boceprevir sind in Tabelle 3 und 4 detailiert aufgeführt.

Therapie-naive Patienten und Relapser

Nicht-Zirrhotiker mit eRVR:

- 12 Wochen TPV / PEG-IFN- $\alpha$ / RBV + 12 Wochen PEG-IFN- $\alpha$ / RBV

Nicht-Zirrhotiker ohne eRVR und alle Zirrhotiker:

- 12 Wochen TPV / PEG-IFN- $\alpha$ / RBV + 36 Wochen PEG-IFN- $\alpha$ / RBV

Partiell- und Null-Responder

Nicht-Zirrhotiker und Zirrhotiker*:

- 12 Wochen TPV / PEG-IFN- $\alpha$ / RBV + 36 Wochen PEG-IFN- $\alpha$ / RBV

- * $\quad$ Bei Null-Responder mit Zirrhose kann ein Lead-In mit PEG-INF- $\alpha$ und RBV evaluiert werden (4 Wochen PEG-IFN- $\alpha$ / RBV, danach TPV nur bei >1 log Stufe Abfall der HCV-RNA nach 4 Wochen)

Tab. 3 Tripletherapie mit Telaprevir (TPV) 
Therapie-naive Patienten

Nicht-Zirrhotiker mit RVR8:

- 4 Wochen PEG-IFN- $\alpha$ / RBV Lead-In

- 24 Wochen BOC / PEG-IFN- $\alpha$ / RBV

Nicht-Zirrhotiker ohne RVR8:

- 4 Wochen PEG-IFN- $\alpha$ / RBV Lead-In

- 24 Wochen BOC / PEG-IFN- $\alpha$ / RBV

- 20 Wochen PEG-IFN- $\alpha$ / RBV

Alle Zirrhotiker:

- 4 Wochen PEG-IFN- $\alpha$ / RBV Lead-In

- 44 Wochen BOC / PEG-IFN- $\alpha$ / RBV

\section{Relapser und Partiell-Responder}

Nicht-Zirrhotiker mit RVR8:

- 4 Wochen PEG-IFN- $\alpha$ / RBV Lead-In

- 32 Wochen BOC / PEG-IFN- $\alpha$ / RBV

Nicht-Zirrhotiker ohne RVR8:

- 4 Wochen PEG-IFN- $\alpha$ / RBV Lead-In

- 32 Wochen BOC / PEG-IFN- $\alpha$ / RBV

- 12 Wochen PEG-IFN- $\alpha$ / RBV

Alle Zirrhotiker:

- 4 Wochen PEG-IFN- $\alpha$ / RBV Lead-In

- 44 Wochen BOC / PEG-IFN- $\alpha$ / RBV

\section{Null-Responder}

Nicht-Zirrhotiker und Zirrhotiker:

- 4 Wochen PEG-IFN- $\alpha$ / RBV Lead-In

- 44 Wochen BOC / PEG-IFN- $\alpha$ / RBV

Tab. 4 Tripletherapie mit Boceprevir (BOC)

Auch bei der Tripletherapie gelten klar definierte Stopp-Regeln $[1 ; 5]$.

Ein Therapieabbruch unter Telaprevir sollte in folgenden Situationen erfolgen:

- HCV-RNA >1000 IU/ml nach 4 oder 12 Wochen Tripletherapie

- HCV-RNA nach 24 Wochen Therapie noch nachweisbar

- HCV-RNA wieder nachweisbar, nachdem sie bereits nicht mehr nachweisbar war (Ausnahme: HCV-RNA blip, d.h. früher transienter Nachweis von HCV-RNA)

Ein Therapieabbruch unter Boceprevir sollte in folgenden Situationen erfolgen:

- HCV-RNA >100 IU $/ \mathrm{ml}$ nach 12 Wochen Tripletherapie

- HCV-RNA nach 24 Wochen Therapie noch nachweisbar

- HCV-RNA wieder nachweisbar, nachdem sie bereits nicht mehr nachweisbar war (Ausnahme: HCV-RNA blip)

- HCV-RNA Abfall $<3$ log in Woche 8

- Bei Patienten mit Zirrhose, bei welchen nach der 4-wöchigen Lead-In-Phase der Abfall der HCV-RNA $<1$ log Stufe liegt (optional)

Auch beim Genotyp 1 sollte bei Null-Respondern oder bei Therapieabbruch aufgrund schwerer Nebenwirkungen oder Resistenzentwicklung ein Therapieversuch mit neuen direkt-antiviralen Medikamenten im Rahmen klinischer Studien erwogen werden bzw. sollte auf die Zulassung neuer Medikamente gewartet werden. 


\section{Neue Therapieoptionen}

Die bereits gut etablierten direkt-antiviralen Medikamente Telaprevir und Boceprevir sind sogenannte NS3/4A Proteaseinhibitoren der 1. Generation. NS3 und NS4A sind nicht-strukturelle Proteine des HC-Virus, welche für die Virusreplikation essentiell sind. Andere für die Virusvermehrung wichtige Virus- und Wirtsproteine, wie zum Beispiel NS5B, NS5A und Cyclophyllin A, sind wichtige Angriffspunkte für die derzeitige Entwicklung neuer antiviraler Medikamente [6].

Die NSB5 Polymerase Inhibitoren werden in 2 Kategorien eingeteilt, in Nukleosidinhibitoren (NI) und Nicht-Nukleosidinhibitoren (NNI). Nls täuschen die natürlich vorkommenden Nukleoside vor und werden in die Virus-RNA integriert, woraufhin die Terminierung der RNA-Bildung induziert wird. NIs haben eine hohe Barriere gegenüber Resistenzen. Die NNIs zeigen eine etwas geringere Wirkung gegenüber dem Genotyp 1 und auch eine raschere Resistenzentwicklung [6]. Ein Beispiel für einen NS5B Nukleosidinhibitor ist Sofosbuvir (GS-7977), welcher gegen alle Genotypen wirksam ist. Die aktuellsten Studien versprechen eine sichere und effektive Therapie. In der FISSION Studie konnten bei den Genotypen 2 und 3 bei einer Therapie nur in Kombination mit RBV ohne Interferon und nur über 12 Wochen Therapiedauer SVR-Raten von 56\% (Genotyp 3) bis 97\% (Genotyp 2) erreicht werden. Die Nebenwirkungen waren signifikant geringer im Vergleich zur Vergleichsgruppe mit PEGINF- $\alpha$ und beinhalteten hauptsächlich Müdigkeit, Übelkeit, Kopfschmerzen und Schlafstörungen. Neutropenie oder Thrombopenie traten in der Interferonfreien Gruppe nicht auf. In der NEUTRINO Studie konnten für die Genotypen 1, 4, 5 und 6 unter einem Therapieregime aus Sofosbuvir, PEGINF- $\alpha$ und RBV über 12 Wochen SVR-Raten von 90\% nachgewiesen werden. Bei Zirrhose-Patienten waren die SVR-Raten sowohl im FISSION Trial mit $47 \%$ als auch im NEUTRINO Trial mit $80 \%$ geringer [7].

Ein Beispiel für einen NSA5-Inhibitor ist Daclatasvir. Auch dieser weist eine hohe antivirale Aktivität gegen verschiedene Genotypen auf, hat aber eine geringere Barriere gegenüber Resistenzen [6].

Ein anderer vielsprechender NSA5-Inhibitor ist Ledipasvir, welcher auch gegen die einzig bekannte HCV-Mutation auf Sofosbuvir (nämlich S282T Mutation) wirksam ist. Ledipasvir wurde in einer Phase2-Studie zusammen mit Sofosbuvir mit und ohne Ribavirin bei Patienten mit Genotyp 1 untersucht (LONESTAR Trial) [8]. Untersucht wurden Therapie-naive Patienten sowie Patienten mit zuvor frustranem Therapieerfolg unter einem Proteaseinhibitor. Bei diesem Interferon-freien Regime lagen die SVR-Raten bei einer Kombination aus Sofosbuvir und Ledipasvir sowohl mit als auch ohne Ribavirin bei sowohl Therapie-naiven als auch Therapie-erfahrenen zwischen 95-100\% nach einer 12 wöchigen Therapiedauer. An Nebenwirkungen traten vor allem Übelkeit, Anämie, Infekte der oberen Atemwege und Kopfschmerzen auf.

Für den Cyclophyllin-Inhibitor Alisporivir konnte ebenfalls eine pan-genotypische Aktivität nachgewiesen werden. Und auch hier zeigten sich effiziente Resultate mit einem Interferon-freien Regime. So konnten bei einer Kombinationstherapie mit Ribavirin bei Patienten mit einer Infektion mit dem Genotyp 2 und 3 SVR-Raten bis zu 71\% nach 24 Wochen nachgewiesen werden [9].

Viele Studien zielen nun auf die Kombination der genannten neuen Medikamente ab. Ziel ist es, ein Interferon-freies Regime (wenn möglich in einer einzelnen Tablette) zu finden mit grossem antiviralem Potential und geringer Rate an Resistenzentwicklungen [11]. 


\section{Management während und nach der Therapie}

Das Therapie-Monitoring beinhaltet einerseits die Überprüfung des Therapieansprechens sowie andererseits die regelmässige Kontrolle hinsichtlich Therapie-Nebenwirkungen.

Das Therapieansprechen unter Therapie mit PEG-INF- $\alpha$ und RBV sowie bei Tripletherapie mit Telaprevir wird durch Messung der HCV-RNA in Woche 4, 12 und 24, am Therapieende und 12, respektive 24 Wochen nach Therapieende bestimmt. Bei Tripletherapie mit Boceprevir erfolgt zusätzlich eine Messung in Woche 8 [1-2].

Die Therapietoxizität sollte nach 1, 2, 4 und dann in 4-8 wöchigen Intervallen überprüft werden. Bei jeder Konsultation sollte der Patient hinsichtlich grippeähnlicher Symptome, Abgeschlagenheit, Depression, Irritabilität, Schlafstörungen, Hautproblemen und Dyspnoe befragt werden. Zur Laboranalyse gehört ein Blutbild (mit Frage nach Anämie, Neutropenie oder Thrombopenie), die Messung der ALT sowie alle 12 Wochen die Messung von TSH [1-2].

Die durch Interferon- $\alpha$ ausgelösten grippeähnlichen Symptome können in der Regel symptomatisch mit Gabe von Paracetamol behandelt werden.

Im Falle eines Aufflammens der entzündlichen Aktivität der Hepatitis mit einem Anstieg der ALT auf das 10-fache der Norm (falls nicht schon zu Therapiebeginn vorhanden) oder im Falle einer schweren bakteriellen Infektion muss die Therapie mit Interferon sofort sistiert werden [1-2].

Bei Entwicklung depressiver Beschwerden hat sich eine Therapie mit Citalopram/Escilatopram bewährt. Auch eine psychologisch bzw. psychiatrische Betreuung sollte dem Patienten zusätzlich empfohlen werden. Bei Patienten mit Depression in der Vorgeschichte und sogar auch bei Patienten ohne bekannte Depression kann eine präventive Gabe von Citalopram/Escilatopram die Entwicklung einer Interferon-induzierten Depression vermindern und somit die Lebensqualität verbessern [10].

Bei Blutbildveränderungen muss je nach Ausprägung die Dosierung von PEG-INF- $\alpha$ und RBV angepasst werden. Gemäss Arzneimittelinformation sollte bei einer Neutropenie von $<750 / \mu$, bei einer Thrombopenie $<50.000 / \mu$ l ebenso wie bei einer ausgeprägten Depression die Dosis von PEG-INF- $\alpha$ $2 \mathrm{a}$ von 180 auf $135 \mu \mathrm{g}$ pro Woche und dann auf $90 \mu \mathrm{g} /$ Woche bzw. PEG-INF- $\alpha$ 2b von 1.5 auf 1.0 und dann auf $0.5 \mu \mathrm{g} / \mathrm{kgKG}$ reduziert werden. PEG-INF-a sollte gestoppt werden bei schwerster Depression, bei Neutropenie von $<500 / \mu$ l oder bei einer Thrombopenie von $<25.000 / \mu l$. Wenn die Neutrophilen bzw. Thrombozyten nach Therapiestopp wieder steigen, kann die Therapie in reduzierter Dosierung wieder begonnen werden. Eine Dosisreduktion von Ribavirin um $200 \mathrm{mg}$ sollte bei Anämie mit einem Hämoglobin von $<100 \mathrm{~g} / \mathrm{l}$ erfolgen. Ribavirin sollte nach Angaben der Arzneimittelinformation gestoppt werden bei einem Hämoglobin von $85 \mathrm{~g} / \mathrm{l}$. Als Alternative kann aber auch Erythropoetin oder eine Bluttransfusion verabreicht werden [1-2].

Unter Tripletherapie mit Telaprevir oder Boceprevir ist die Entwicklung einer Anämie häufiger und oft ausgeprägter. Bluttransfusion oder Gabe von Erythropoetin sind hier neben der Dosisreduktion von Ribavirin häufiger notwendig [5].

Telaprevir kann zudem Übelkeit, Diarrhoe, anorektale Beschwerden sowie Hautausschläge, Juckreiz und in seltenen Fällen schwerwiegende Hautmanifestationen wie ein DRESS- (drug related eosinophilia with systemic symptoms) oder ein Stevens-Johnson-Syndrom verursachen. Die Hautausschläge unter Telaprevir gleichen denen unter alleiniger PEG-INF- $\alpha$ /RBV -Therapie, sind aber häufig schwerer. Bei schwerem Rash sowie Verdacht auf DRESS- oder Stevens-JohnsonSyndrom muss die Therapie mit Telaprevir sofort gestoppt werden und ein dermatologisches Konsil erfolgen [5].

Boceprevir kann neben ausgeprägter Anämie zu Neutropenie, Müdigkeit und Geschmacksstörungen führen.

Bei Therapie mit Boceprevir und Telaprevir ist zudem das hohe Potential an Arzneimittelinteraktionen zu beachten, da beide Medikamente über Cytochrom P450 (CYP 3A4) metabolisiert werden. Kontraindiziert ist zum Beispiel die gleichzeitige Gabe von Telaprevir oder Boceprevir zusammen mit Atorvastatin, Lovastatin, Simvastatin, Sildenafil, Amiodaron, Carbamazepin, Phenytoin, Johanniskraut, oralem Midazolam oder Domperidon. In Online-Datenbanken (z.B. unter www.hepdruginteractions.org) können erlaubte bzw. kontrainzidierte Medikamente recherchiert werden [5].

Tabelle 5 zeigt in einer Übersicht häufige Nebenwirkungen sowie deren Management. 


\begin{tabular}{|c|c|c|}
\hline Nebenwirkung & Ursache & Management \\
\hline Grippeähnliche Symptome & PEG-IFN- $\alpha$ & $\begin{array}{l}\text { Paracetamol } \\
\text { (ggf. auch prophylaktisch) }\end{array}$ \\
\hline Depression & PEG-IFN- $\alpha$, Boceprevir & $\begin{array}{l}\text { Psychologisch/psychiatrische } \\
\text { Betreuung, } \\
\text { Citalopram/Escilatopram }\end{array}$ \\
\hline Anämie & Ribavirin, Telaprevir, Boceprevir & $\begin{array}{l}\text { Dosisreduktion von Ribavirin, } \\
\text { Erythropoetin-Gabe, } \\
\text { Transfusion }\end{array}$ \\
\hline Neutropenie & PEG-IFN- $\alpha$, Boceprevir & $\begin{array}{l}\text { Dosisreduktion von PEG-IFN- } \alpha \\
\text { bei Neutrophilen }<750 / \mu \mathrm{l}\end{array}$ \\
\hline & & $\begin{array}{l}\text { Stopp von Peg-IFN bei } \\
\text { Neutrophilen }<500 / \mu \mathrm{l}\end{array}$ \\
\hline Thrombopenie & $\begin{array}{l}\text { PEG-IFN- } \alpha \text {,Telaprevir, } \\
\text { Boceprevir }\end{array}$ & $\begin{array}{l}\text { Dosisreduktion von PEG-IFN- } \alpha \\
\text { bei Thrombozyten }<50.000 / \mu l\end{array}$ \\
\hline & & $\begin{array}{l}\text { Stopp von PEG-IFN- } \alpha \text { bei } \\
\text { Thrombozyten }<25.000 / \mu 1\end{array}$ \\
\hline $\begin{array}{l}\text { Hautausschlag,Juckreiz, } \\
\text { Hauttrockenheit }\end{array}$ & Ribavirin, Telaprevir & $\begin{array}{l}\text { Therapiestopp bei schwerem } \\
\text { Rash, sonst topische Therapie, } \\
\text { z.B. Excipial Lipolotio®, evtl. in } \\
\text { Kombination mit topischen } \\
\text { Steroiden und Antihistaminika }\end{array}$ \\
\hline Übelkeit & Telaprevir & $\begin{array}{l}\text { Metoclopramid, } \\
\text { KEIN Domperidon }\end{array}$ \\
\hline $\begin{array}{l}\text { Proktalgie,Hämorrhoiden, } \\
\text { analer Pruritus }\end{array}$ & Telaprevir & $\begin{array}{l}\text { Lokaltherapie z.B. mit Procto- } \\
\text { Glyvenol } \AA\end{array}$ \\
\hline $\begin{array}{l}\text { Autoimmunthyreoiditis, } \\
\text { Hypothyreose }\end{array}$ & $\begin{array}{l}\text { PEG-IFN- } \alpha \text {,Telaprevir, } \\
\text { Boceprevir }\end{array}$ & $\begin{array}{l}\text { Therapiestopp bei Autoimmun- } \\
\text { Thyreoiditis }\end{array}$ \\
\hline Autoimmunhepatitis & PEG-IFN- $\alpha$, Telaprevir & Therapiestopp \\
\hline Erniedrigte Krampfschwelle & PEG-IFN- $\alpha$ & Konsil durch Neurologe \\
\hline Teratogenität & Ribavirin & $\begin{array}{l}\text { Kontrazeption während bis } 6 \\
\text { Monate nach Therapie }\end{array}$ \\
\hline
\end{tabular}

Tab 5. Häufige Nebenwirkungen und ihr Management

Nach Therapieende muss das Monitoring des Patienten weiter fortgesetzt werden. Bei Patienten ohne Leberzirrhose, die durch die Therapie eine SVR erreicht haben, sollten nach 48 Wochen und nochmal 1 Jahr danach die ALT und HCV-RNA bestimmt werden. Ist die ALT weiterhin normwertig und die HCV-RNA nicht nachweisbar, gilt der Patient als geheilt. Auch TSH und die Schilddrüsenhormone sollten 1 Jahr nach Therapieabschluss nochmals gemessen werden, da eine therapieinduzierte Hypothyreose auch nach Therapieende noch auftreten kann. Bei Patienten mit Zirrhose muss ein unlimitiertes regelmässiges Monitoring erfolgen mit Überwachung hinsichtlich Ösophagusvarizen alle 2-3 Jahre sowie hinsichtlich HCC mittels Abdomensonographie und Bestimmung von a-Fetoprotein alle 6 Monate [2]. 
Abschliessend sind in Tabelle 6 die Kosten der derzeitigen Therapien gegenübergestellt.

\begin{tabular}{|c|c|c|}
\hline Medikament & Kosten pro Monat & $\begin{array}{l}\text { Kosten für jeweils kürzeste } \\
\text { bzw. längste Therapiedauer }\end{array}$ \\
\hline PEG-IFN- $\alpha$ & $1 ‘ 113 \mathrm{CHF}$ & 6’678 bzw.13’356 CHF \\
\hline RBV & $978-1 ‘ 173 \mathrm{CHF}^{2}$ & $\begin{array}{l}5^{\prime} 868-7^{\prime} 038 \mathrm{CHF}^{2} \text { bzw. } \\
11^{\prime} 736-14^{\prime} 076 \mathrm{CHF}^{2}\end{array}$ \\
\hline Victrelis $®$ & $4.433 \mathrm{CHF}$ & 26'598 bzw. 48'763 CHF \\
\hline Incivo@ & $11 ' 846 \mathrm{CHF}$ & $35^{\prime} 540 \mathrm{CHF}$ \\
\hline
\end{tabular}

Tab. 6 Therapiekosten im Vergleich. Die Kosten wurden aus dem compendium.ch® entnommen.

${ }^{1} \mathrm{Da}$ Telaprevir (Incivo®) immer nur über 12 Wochen verabreicht wird, ist die Summe der Kosten von Incivo® auf 12 Wochen hochgerechnet. Bei Victrelis ${ }^{\circledR}$ sind die Kosten auf die minimale Gabe von 24 bzw. die maximale Gabe von 44 Wochen aufsummiert. Bei PEG-INF- $\alpha$ und RBV sind die Kosten für 24 bzw. 48 Wochen angegeben.

${ }^{2}$ Die Dosis und entsprechend die Kosten von RBV variieren je nach Körpergewicht (< bzw. $>75 \mathrm{~kg}$ ). 
Im Alter von 29 Jahren stellte sich der Patient zur Blutspende zur Verfügung. Hier wurde im Rahmen der Screeningtests eine Hepatitis C mit dem Genotyp 1b diagnostiziert. Ausser einer chronischen Müdigkeit hatte der Patient bis zu diesem Zeitpunkt keine Beschwerden beklagt. Intravenöser Drogenkonsum, Bluttransfusionen, sexuelle Risikokontakte oder eine Hepatitis C in der Familie wurden verneint. Die Ursache der Ansteckung blieb unklar.

In der Folge wurde eine Mono-Therapie mit Interferon durchgeführt, bei fehlendem Ansprechen aber wieder abgebrochen. 10 Jahre später erfolgte ein erneuter Therapieversuch, diesmal mit einer Kombinationstherapie mit PEG-INF- $a$ und RBV. Auch hier blieb der Erfolg aus und die Therapie wurde sistiert. An Nebenwirkungen beklagte der Patient unter den genannten Therapieregimen lediglich grippeähnliche Symptome sowie Stimmungsschwankungen, welche als nur wenig belastend empfunden wurden.

Im Alter von 41 Jahren wurde histologisch das Vorliegen einer Leberzirrhose diagnostiziert. Im Verlauf stellte sich der Patient schliesslich mit Blut im Stuhl erneut ärztlich vor. Als Ursache fanden sich Ösophagusvarizen im Rahmen der Leberzirrhose. Nach Varizenligatur wurde der Entscheid zu einem Therapieversuch der Hepatitis C mit einer Tripletherapie mit PEG-IFN- $\alpha$, RBV und Telaprevir gefällt.

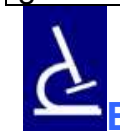

Status

44-jähriger, wacher Patient, allseits orientiert, in gutem AZ und adipösem EZ (BMI $38,6 \mathrm{~m}^{2} / \mathrm{kg}$ ), normoton, normokard, afebril. Blasses Integument. Abdomen weich, indolent, keine Organomegalien palpabel, unauffälliger Herz-Lungen-Befund und Gefässtatus. Keine Ödeme.

Aktuelle Therapie

Pegasys $\AA$ (Peg-Interferon alpha 2a) 180 $\mu$ g 1x/Woche s.c.

Copegus $\AA$ (Ribavirin) $200 \mathrm{mg}$ p.o. 3-0-3

Incivo $\AA^{8}$ (Telaprevir) $375 \mathrm{mg}$ p.o. alle 8 Stunden 2 Tabletten mit $20 \mathrm{~g}$ Fett

Routinelabor vor Beginn der Tripletherapie

Hämoglobin $89 \mathrm{~g} / \mathbf{l} \downarrow$, Thrombozyten $126 \mathrm{G} / \mathbf{l} \downarrow$, Bereits vor Therapiebeginn lag bei dem Leukozyten $3.23 \mathrm{G} / \mathrm{l}$, INR 1.2, ALT $31 \mathrm{U} / \mathrm{l}$, AST $49 \mathrm{U} / \mathrm{l}$, Patienten eine Anämie bei oberer Gl-Blutung GGT $45 \mathrm{U} / \mathrm{l}$, alk. Phosphatase $78 \mathrm{U} / \mathrm{l}$, Bilirubin 8 im Rahmen von Ösophagusvarizen vor.
Typischerweise manifestiert sich die chronische Hepatitis C oligo- oder asymptomatisch. Müdigkeit als unspezifisches Symptom ist häufig. Die Transmission der Infektion ist insbesondere bei Infektion vor 1990 häufig auf Medizinprodukte zurückzuführen, da zu diesem Zeitpunkt noch keine Screeningtests von Blut- und Medizinprodukten etabliert waren. Nicht immer kann die Ursache der Ansteckung gefunden werden.

Die in der Vergangenheit gängige Therapie der chronischen Hepatitis $\mathrm{C}$ war die Gabe von Interferon-a. Im Verlauf wurde diese von einer Kombinationstherapie mit Ribavirin abgelöst. Der Patient hat die typischen

Nebenwirkungen, die bei der Mehrheit der behandelten Patienten auftreten, erlebt. Hier stehen vor allem grippeähnliche Symptome im Vordergrund. Auch psychische Beschwerden mit Depression oder Dysthymie sind häufig. Innerhalb von 2 Jahrzehnten entwickeln bis zu $20 \%$ der chronisch Infizierten eine Leberzirrhose wie auch dieser Patient. Eine kompensierte Leberzirrhose stellt keine Kontraindikation für eine Therapie dar. Beim Genotyp 1 ist beim Fehlen von Kontraindikationen eine Tripletherapie noch derzeitiger Standard.

\section{Kommentar}

Bei dem Patienten lag eine Adipositas Grad II vor. Das Vorliegen einer Adipositas bei chronischer Hepatitis C geht mit einer erhöhten Progressionsrate zur Fibrose und Zirrhose einher.

Wichtig bei der Einnahme der NS3/A4 Proteaseinhibitoren ist eine strikte Einhaltung der Einnahmezeiten alle 8 Stunden $( \pm 1$ Stunde) zur Minimierung einer Resistenzentwicklung. Telaprevir muss zur Verbesserung der Resorption mit $20 \mathrm{~g}$ Fett eingenommen werden.

im Rahmen von Ösophagusvarizen vor.




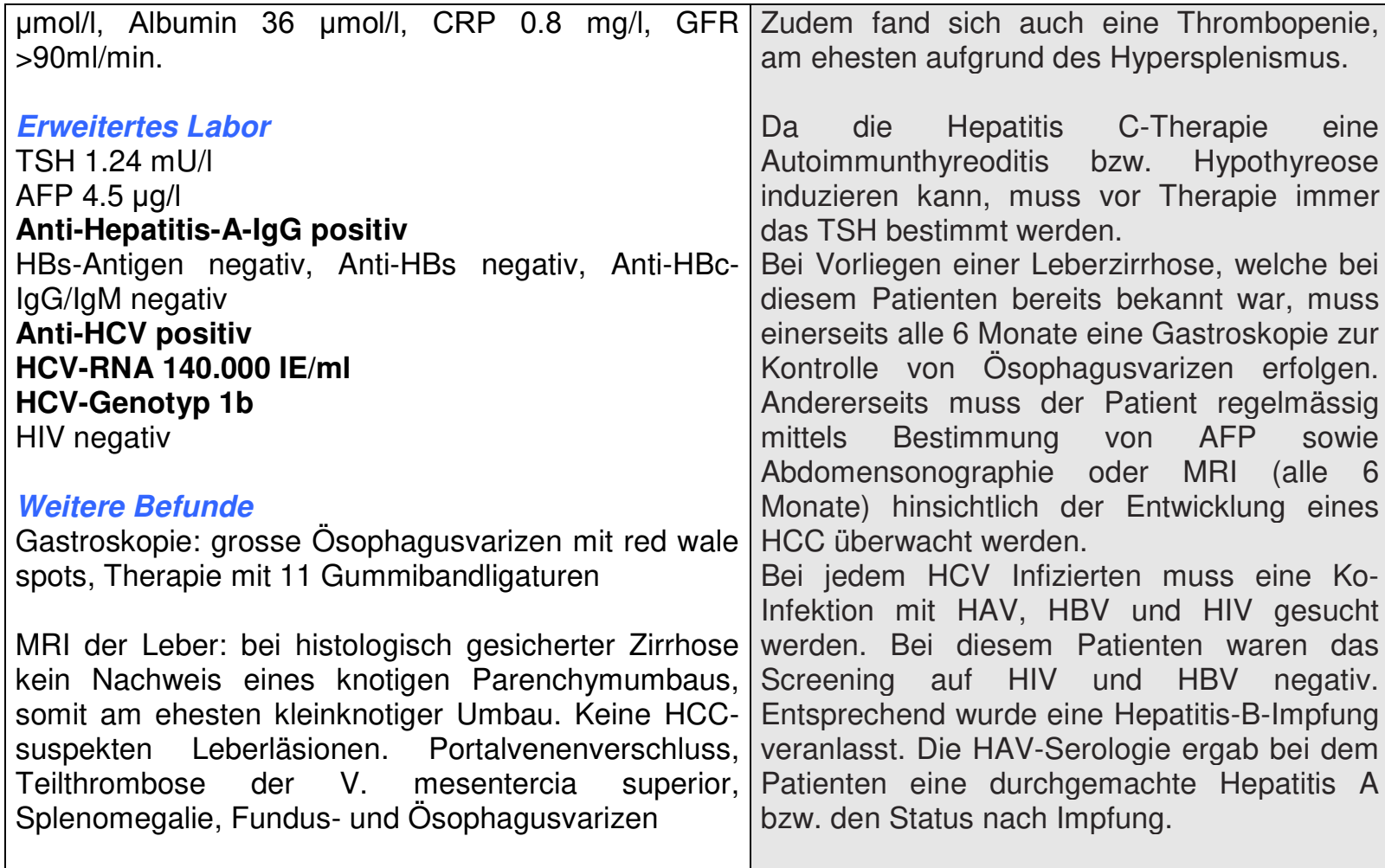

I.

In der Kontrolle in Therapiewoche (TW) 1 beklagte der Patient bereits Schüttelfrost, Fieber und starke Müdigkeit, welche sich durch die gesamte Therapiedauer hindurch zog. Zur symptomatischen Therapie wurde Paracetamol (max. $3 \times 1 \mathrm{~g}$ ) verschrieben. Auch deutliche psychische Veränderungen mit erhöhter Reizbarkeit, depressiver Stimmungslage und gestörtem Schlaf-Wach-Rhythmus kamen hinzu. Der Patient suchte sich hier Unterstützung bei einem Psychologen, eine medikamentöse Therapie wurde nicht gewünscht. Als zusätzliche Nebenwirkung kam es im Verlauf zu einer ausgeprägten Anämie mit Hb-Werten bis $63 \mathrm{~g} / \mathrm{l}$, woraufhin die Dosis von Ribavirin mehrfach reduziert werden musste und schliesslich auch einmalig eine Bluttransfusion erfolgte. Hiernach blieb der Hämoglobinwert stabil. Auch die Thrombozyten sanken auf bis zu 50-60 G/l, blieben aber in diesem Bereich stabil. Zudem entwickelte sich eine Hyperbilirubinämie. Als typische Nebenwirkung von Telaprevir traten Hauttrockenheit und Pruritus auf, welche mittels Excipial Lipolotio $\circledast$ sowie Xyzal® symptomatisch behandelt wurden. Der Patient erlebte einen Gewichtsverlust von insgesamt $30 \mathrm{~kg}$ in 7 Monaten, teils als bekannte Nebenwirkung, teils auch durch strikte Diät des Patienten.

In TW4 und TW12 war die HCV-RNA erfreulicherweise nicht mehr nachweisbar, entsprechend einer extended rapid virological response (eRVR). Die Therapie wurde nach 12 Wochen mit PEG-INF- $\alpha$ und RBV alleine weitergeführt und schliesslich nach 50 Wochen abgeschlossen. Auch bei Therapieende war keine HCV-RNA mehr nachweisbar. 


\section{Fragen zur Therapie der chronischen Hepatitis C}

\section{Frage 1}

Um wieviel Prozent kann die SVR-Rate bei Tripletherapie mit Telaprevir oder Boceprevir beim Genotyp 1 bei Therapie-naiven Patienten verbessert werden? (Einfachauswahl)
a) $25 \%$
b) $50 \%$
c) $95 \%$
d) $70 \%$
e) $5 \%$

\section{Frage 2}

Welche Nebenwirkung tritt bei Tripletherapie nicht vermehrt auf? (Einfachauswahl)
a) Anämie
b) Hautveränderungen
c) Gastrointestinale Beschwerden
d) Thromboembolien

\section{Frage 3}

Die gleichzeitige Einnahme von Proteaseinhibitoren der 1. Generation und Atorvastatin sollte vermieden werden,

weil

die renale Ausscheidung dieser Proteaseinhibitoren dadurch erhöht wird.
a) Aussage 1 und 2 sind richtig
b) Nur Aussage 1 ist richtig
c) Nur Aussage 2 ist richtig
d) Beide Aussagen sind falsch

\section{Frage 4}

Welche Aussage ist falsch? (Einfachauswahl)

a) Sofosbuvir zeigt gegen alle HCV-Genotypen einen antiviralen Effekt.

b) Während und 6 Monate nach Therapie mit Ribavirin sollte eine Schwangerschaft verhütet werden.

c) Bei Tripletherapie und schwerer Anämie dürfen PEG-IFN- $\alpha 2 \mathrm{a} / \mathrm{b}$ und RBV zwar in gleicher Dosis weitergegeben werden, die Dosis des Proteaseinhibitors muss aber reduziert werden.

d) Bei Tripletherapie mit Telaprevir kann bei Übelkeit eine symptomatische Therapie mit Metoclopramid durchgeführt werden.

\section{Frage 5}

Folgende Aussage zur umfassenden Aufklärung bei chronischer Hepatitis $C$ ist falsch? (Einfachauswahl)
a) Nach Diagnosestellung muss beim Geschlechtsverkehr bei langjährigen Sexualpartnern ab sofort verhütet werden.
b) Nach Diagnosestellung sollte keine gemeinsame Zahnbürste verwendet werden.
c) Eine Gewichtsreduktion bei Adipositas hat einen positiven Einfluss auf das Risiko der Entwicklung einer Fibrose und Zirrhose bei chronischer Hepatitis C.
d) Eine Impfung gegen Hepatitis A und Hepatitis B sollte bei negativen Serologien sobald wie möglich erfolgen.




\begin{abstract}
Autoren
Dr. med. Stella Schaffstein, Klinik und Poliklinik für Innere Medizin, Universitätsspital Zürich

Dr. med. Silvana K. Rampini, Klinik und Poliklinik für Innere Medizin, Universitätsspital Zürich

Prof. Dr. med. Beat Müllhaupt, Klinik für Gastroenterologie und Hepatologie, Universitätsspital Zürich
\end{abstract}

\title{
Korrespondenzadresse
}

Dr. med. Silvana K. Rampini

Klinik und Poliklinik für Innere Medizin

UniversitätsSpital Zürich

Rämistrasse 100

$\mathrm{CH}-8091$ Zürich

Silvana.rampini@usz.ch

\section{Bibliographie}

1. European Association for the Study of the Liver. EASL Clinical Practice Guidelines: Management of hepatitis C virus infection. Journal of Hepatology. 2013;168-8278(13)00794-0

2. European Association for the Study of the Liver. EASL Clinical Practice Guidelines: Management of hepatitis C virus infection. Journal of Hepatology. 2011;55:245-64

3. Tahan $\mathrm{V}$ et al. Sexual transmission of HCV between spouses. Am J Gastroenterol 2005;100:821-824

4. Arshad M, El-Kamary SS, Jhaveri R. Hepatitis C virus infection during pregnancy and the newborn period - are they opportunities for treatment? J Viral Hepat. 2011 Apr;18:229-36

5. Treatment of chronic hepatitis C genotyp 1 with triple therapy comprising telaprevir or boceprevir. Swiss Med Wkly. 2012;142:w13516

6. Alexopoulou A, Papatheodoridis GV. Current progress in the treatment of chronic hepatitis C. World J Gastroenterology. 2012. 14;18 (42): 6060-6069

7. Lawitz $E$ et al. Sofosbuvir for previously untreated chronic hepatitis C infection. NEJM 2013. May 16:1878-1887

8. Lawitz $\mathrm{E}$ et al. Sofosbuvir and ledipasvir fixed-dose combination with and without ribavirin in treatment-naive and previously treated patients with genotype 1 hepatitis $C$ virus infection (LONESTAR): an open-label, randomised, phase 2 trial. Lancet. 2013 Nov 1. 62121-2

9. Guedj $\mathrm{J}$ et al. Modeling viral kinetics and treatment outcome during alisporivir interferon-free treatment in HCV genotype 2/3 patients. Hepatology. 2013 Dec 23. doi: 10.1002/hep.26989

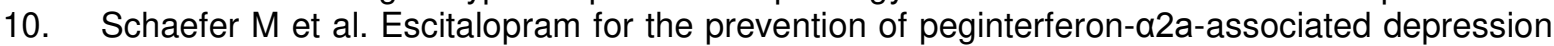
in hepatitis $C$ virus-infected patients without previous psychiatric disease: a randomized trial. Ann Intern Med. 2012 Jul 17;157:94-103

11. Kowdley KV et al. Phase $2 b$ trial of interferon-free therapy for hepatitis $C$ virus genotype $1 . \mathrm{N}$ Engl J Med. 2014 Jan 16; 370:222-32 


\section{Antworten zu den Fragen zur Therapie der chronischen Hepatitis C}

\section{aus PRAXIS Nr. XY}

\section{Frage 1}

\section{Richtig ist Antwort a)}

Durch eine Tripletherapie mit Telaprevir oder Boceprevir zusammen mit PEG-IFN- $\alpha$ und RBV konnten die SVR-Raten um 25\% gesteigert werden, somit ist Antwort a) richtig. Die SVR-Raten liegen damit bei Therapie-naiven bei $>70 \%$, bei Relapsern bei $>80 \%$, bei Partiell-Respondern bei $50 \%$ und bei vorherigen Null-Respondern bei $30 \%$.

\section{Frage 2}

\section{Richtig ist Antwort d)}

Unter Tripletherapie kann eine schwerwiegende Anämie auftreten. Nebenwirkungen der Haut treten unter Telaprevir ebenfalls auf, diese reichen von Hauttrockenheit und Juckreiz bis hin zu schweren allergischen Reaktionen (wenn auch sehr selten) mit Stevens-Johnson-Syndrom oder DRESS (drug related eosinophilia with systemic symptoms)-Syndrom.

Insbesondere Telaprevir verursacht gastrointestinale Beschwerden wie Übelkeit, Diarrhoe und anorektale Beschwerden. Vermehrte thromboembolische Ereignisse wurden nicht beschrieben, Antwort d) ist somit falsch.

\section{Frage 3}

\section{Richtig ist Antwort b)}

Die gleichzeitige Einnahme von Telaprevir oder Boceprevir zusammen mit Atorvastatin sollte vermieden werden (Aussage 1 ist richtig), da beide über CYP 3A4 metabolisiert werden und somit die Atorvastatin-Exposition deutlich erhöht sein kann. Boceprevir wird vorwiegend hepatisch eliminiert und Atorvastatin beeinflusst die renale Ausscheidung nicht. Somit ist Aussage 2 falsch.

\section{Frage 4}

\section{Richtig ist Antwort c)}

Alle Aussagen sind richtig, bis auf Aussage c). Bei schwerer Anämie unter Tripletherapie sollte die Dosis von Ribavirin reduziert werden. Eine zusätzliche Option ist die Gabe von Erythropoetin oder eine Bluttransfusion. Die Dosis des Proteaseinhibitors sollte nie verändert werden, um die Entwicklung von Resistenzen zu vermeiden.

\section{Frage 5}

\section{Richtig ist Antwort a)}

Alle Aussagen sind richtig, bis auf Aussage a). Da das sexuelle Übertragungsrisiko bei chronischer Hepatitis C (abgesehen von Analverkehr) sehr gering ist, muss bei langjährigen Sexualpartnern nicht zwingend mit Kondomen verhütet werden. 